Shinji Kondoh · Hirobumi Sugawara • Naoki Harada

Naomichi Matsumoto - Hirofumi Ohashi - Masato Sato

Piranit N. Kantaputra - Toshihiko Ogino $\cdot$ Hiroaki Tomita

Tohru Ohta • Tatsuya Kishino • Yoshimitsu Fukushima

Norio Niikawa $\cdot$ Koh-ichiro Yoshiura

\title{
A novel gene is disrupted at a 14q13 breakpoint of $t(2 ; 14)$ in a patient with mirror-image polydactyly of hands and feet
}

Received: October 29, 2001 / Accepted: December 27, 2001

\begin{abstract}
Mirror-image polydactyly of hands and feet (MIP) is a very rare congenital anomaly characterized by mirror-image duplication of digits. To isolate the gene responsible for MIP, we performed translocation breakpoint cloning from an MIP patient with $\mathrm{t}(2 ; 14)(\mathrm{p} 23.3 ; \mathrm{q} 13)$. We isolated a good candidate gene for MIP that was disrupted by the translocation of the patient. We had previously constructed a 1.2-megabase bacterial artificial chromosome (BAC)/P1-derived artificial chromosome (PAC) contig covering the $14 q 13$ breakpoint of $\mathrm{t}(2 ; 14)(\mathrm{p} 23.3 ; \mathrm{q} 13)$. From a $500-\mathrm{kb}$ segment consisting of seven BAC/PAC clones in the contig, we isolated a novel gene (the mirror-image polydactyly 1 gene, designated as MIPOL1, GenBank Accession No. AY059470), in addition to the hepatocyte nuclear fac-
\end{abstract}

S. Kondoh $(\square) \cdot$ H. Sugawara $\cdot$ N. Harada $\cdot$ N. Matsumoto

H. Tomita $\cdot$ N. Niikawa $\cdot$ K-i. Yoshiura

Department of Human Genetics, Nagasaki University School of

Medicine, 1-12-4 Sakamoto, Nagasaki 852-8523, Japan

Tel. +81-95-849-7119; Fax +81-95-849-7121

e-mail:kyoshi@net.nagasaki-u.ac.jp

H. Sugawara $\cdot$ N. Matsumoto $\cdot$ H. Ohashi $\cdot$ H. Tomita .

T. Kishino $\cdot$ Y. Fukushima $\cdot$ N. Niikawa $\cdot$ K.-i. Yoshiura

CREST, Japan Science and Technology Corporation, Kawaguchi,

Japan

H. Sugawara $\cdot$ T. Ogino

Department of Orthopedic Surgery, Yamagata University School of Medicine, Yamagata, Japan

H. Ohashi

Division of Medical Genetics, Saitama Children's Medical Center, Saitama, Japan

M. Sato

Division of Orthopedics, Saitama Children's Medical Center,

Saitama, Japan

P.N. Kantaputra

Department of Pediatric Dentistry, Faculty of Dentistry, Chiang Mai

University, Chiang Mai, Thailand

T. Ohta $\cdot$ T. Kishino

Nagasaki University Gene Research Center, Nagasaki, Japan

Y. Fukushima

Department of Hygiene and Medical Genetics, Shinshu University

School of Medicine, Matsumoto, Japan tor 3 alpha gene (HNF3A, GenBank Accession No. XM 007360). MIPOL1 spans about $350 \mathrm{~kb}$, comprises 15 exons, and encodes 442 amino acids. Northern blot analysis revealed that MIPOL1 expression is definite but very weak in adult heart, liver, skeletal muscle, kidney, and pancreas, and in fetal kidney. In view of the genome sequence and the contig map constructed, the $14 \mathrm{q} 13$ breakpoint of the patient was identified as located in intron 11 of MIPOL1, indicating that the gene was disrupted by the translocation, and that the breakage resulted in MIPOL1 protein truncation. Whole-mount in situ hybridization in mouse resulted in mouse Mipoll signals all over E10.5-E13.5 mouse embryos. Two other unrelated patients with limb anomalies similar to MIP were subjected to mutation analysis of MIPOL1, but none had any mutations. We then isolated BAC clones from the other breakpoint, 2p23.3. A search for genes and expressed sequence tags in a more than $300-\mathrm{kb}$ region around the $2 \mathrm{p} 23.3$ breakpoint found only the neuroblastoma-amplified protein gene (NAG, GenBank Accession No. NM 015909), which is located at least $50 \mathrm{~kb}$ centromeric to the breakpoint and is not likely to be related to MIP. MIPOL1 is a good candidate gene for the MIP type of anomaly.

Key words Mirror-image polydactyly · Translocation $\mathrm{t}(2 ; 14)(\mathrm{p} 23.3 ; \mathrm{q} 13) \cdot$ Breakpoint cloning $\cdot 14 \mathrm{q} 13$ Breakpoint $\cdot$ Novel gene $\cdot M I P O L 1 \cdot$ Coiled-coil domain

\section{Introduction}

Mirror-image polydactyly of hands and feet (MIP) is a very rare congenital anomaly characterized by mirror-image duplication of digits. It is occasionally associated with dimelia of the ulnae and fibulae, tibial and/or fibular hypoplasia, nasal abnormality, and other malformations. Most cases are sporadic, but parent-child transmissions observed in familial cases suggest an autosomal dominant mode of inheritance (Laurin et al. 1964; Kogekar et al. 1993; Martin et al. 1993; Martinez-Frias et al. 1994; Hersh et al. 1995; 
Hatchwell and Dennis 1996). We previously reported a Japanese boy with postaxial MIP without any other anomalies (Ohashi et al. 1995; Kim et al. 1997). Since his limb anomaly was associated with a de novo balanced chromosomal translocation, $\mathrm{t}(2 ; 14)(\mathrm{p} 23.3 ; \mathrm{q} 13)$, we assumed that the translocation might have affected the function of a putative gene that might play a role in limb development (Matsumoto et al. 1997a;b). We also had previously constructed a 1.2-Mb BAC/PAC contig covering the $14 \mathrm{q} 13$ breakpoint of the patient (Matsumoto et al. 1997b). Here, we report on a novel gene that is disrupted at the $14 \mathrm{q} 13$ breakpoint.

\section{Materials and methods}

Isolation of transcribed sequences from the $14 \mathrm{q} 13$ breakpoint region

We attempted to isolate transcribed sequences from a 500-kb segment between markers AFM200ZH4 and P13T7 (Fig. 1a) within the previously constructed $1.2-\mathrm{Mb}$ bacterial artificial chromosome (BAC)/p1-derived artificial chromosome (PAC) contig (Matsumoto et al. 1997b). To isolate $\mathrm{CpG}$ island-rich sequences, DNA from seven BAC/PAC clones (B319, B305, B368, P29, P7, P163, and P464) in the segment was double-digested with PstI/SacI, PstI/BssHII, or PstI/EagI, and the digests were subcloned into pBluescriptII SK(-) (Stratagene, La Jolla, CA USA). In parallel, DNA from all clones but B368 was sonicated, treated with Mung-bean nuclease (New England Biolabs, Beverly, MA, USA), and cloned into pBluescriptII SK(-). For exon trapping, after DNA was partially digested with Sau3AI, large fragments $(>2 \mathrm{~kb})$ were extracted from agarose gel and cloned into pSPL3 according to the manufacturer's protocol (Gibco BRL, Carlsbad, CA, USA). DNA was subjected to sequencing with an autosequencer (model 377, PE Applied Biosystems, Foster City, CA, USA) by using a BigDye terminator sequencing kit (PE Applied Biosystems). The trapped sequences were analyzed with a BLAST search, and genomic sequences around the putative exons were subjected to the Grail II program. The BLAST search was performed against the expressed sequence tag (EST) and the genomic sequence databases.

Primers were designed for putative exons, and cDNA was amplified by $3^{\prime}$ - and $5^{\prime}$-rapid amplification of cDNA ends (RACE) from human muscle and brain Marathon cDNA libraries (Clontech, Palo Alto, CA, USA). IMAGE cDNA clones matching the obtained sequence were subjected to sequence analysis. Exon-intron boundaries were confirmed by comparing the cDNA and genomic sequences.

\section{Northern blot analysis}

To confirm the expression of the newly isolated cDNA (hereafter designated MIPOL1), northern blot analysis was carried out. A 313-bp DNA segment was amplified by polymerase chain reaction (PCR) from the IMAGE clone using the primers Mipol1sense, 5'-TGACATGACATTACAGG AAT-3'; and Mipol1antis, 5'-TAGTAAACTCGAAGGG TC- $3^{\prime}$, and used as a probe for exons 12 to 15 of MIPOL1. The probe was radiolabeled by a random primer labeling kit (Amersham, Buckinghamshire, UK) and hybridized at $60^{\circ} \mathrm{C}$ for $4 \mathrm{~h}$ in the HybriExpress (Clontech), and then washed at $55^{\circ} \mathrm{C}$ in $0.2 \mathrm{xSC} / 0.1 \%$ sodium dodecyl sulfate (SDS). Autoradiography was performed at $-80^{\circ} \mathrm{C}$ for 4 weeks. An oligonucleotide synthesized from the $\beta$-actin gene was used as a control probe.

Whole-mount in situ hybridization in the mouse

The mouse Mipoll cDNA was obtained by reverse transcriptase (RT)-PCR of total RNA from the mouse embryo. The RT-PCR product was cloned into a pT7T3 $\alpha-19 /$ SmaI vector and used as a probe for whole-mount in situ hybridization of E10.5-E13.5 mouse embryos. The probe contained Mipoll exons 14 and 15 that showed 85\% homology
Fig. 1. Genomic organization of the mirror-image polydactyly 1 (MIPOL1) gene. The MIPOL1 gene is about $350 \mathrm{~kb}$ long, consists of 15 exons, and is disrupted at exon 11 by the translocation. The breakpoint is indicated by the vertical arrow at the top of figure. Breakage has occurred between STS markers P29SP6 and B305T7. The MIPOL1 gene is transcribed from centromer to telomere, while the $H N F 3 A$ gene is transcribed from telomere to centromere. CENT, centromere; TEL, Telomere

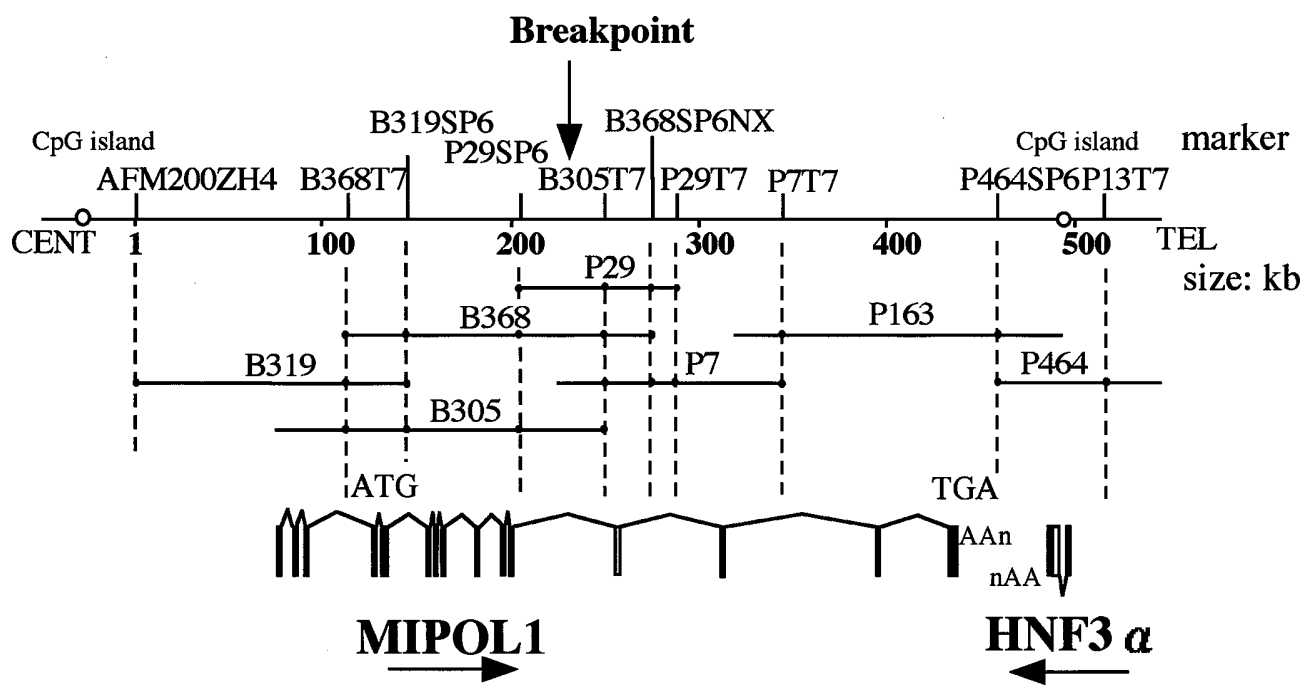


with those of the human MIPOL1 gene. The in situ hybridization procedure was performed as described previously (Yoshiura et al. 1998).

Mutation analysis in patients with mirror-image polydactyly

Two unrelated, karyotypically normal patients, a Japanese boy with postaxial mirror-image polydactyly of the hands, and a Thai man with Laurin-Sandrow syndrome (Kantaputra 2001) were studied for the MIPOL1 mutation as described previously (Kinoshita et al. 2000). Both disorders are clinically similar to MIP. PCR-direct sequencing was performed using 11 primer sets designed to amplify exons 5-15 of MIPOL1 and their exon-intron boundaries. PCR (35 cycles) was done with a GeneAmp 9600 system (Perkin Elmer, Shelton, CT, USA) under the following conditions: denaturation at $94^{\circ} \mathrm{C}$ for $30 \mathrm{~s}$, annealing at $50^{\circ} \mathrm{C}$ for $30 \mathrm{~s}$, and extension at $72^{\circ} \mathrm{C}$ for $30 \mathrm{~s}$. After purification of the PCR product with a QIAquick PCR Purification Kit (Qiagen, Valencia, CA, USA), sequencing was carried out using the BigDye terminator cycle sequencing reaction kit (PE Applied Biosystems) and a Prism 377 DNA sequencer (PE Applied Biosystems).

Analysis of another breakpoint at 2p23.3

A fluorescent in situ hybridization (FISH) analysis was performed using BAC clones mapped to chromosome 2 as probes to search for the 2 p23.3 breakpoint-covering clones, as described previously (Matsumoto et al. 1997b).

\section{Results}

Isolation of a novel gene, MIPOL1, and its protein showing weak homology to CARD14

With the CpG-island sequencing strategy, we detected only one transcribed sequence, which corresponded to the hepatocyte nuclear factor 3 alpha gene $(H N F 3 A)$, within the $500-\mathrm{kb}$ contig analyzed (Fig. 1). We also obtained some other sequences predicted to be exons with the random sequencing method. Two exons (exons 10 and 11) were isolated from the BAC B386 clone that showed split FISH signals on the patient's chromosome (Ohashi et al. 1995; Kim et al. 1997), and they matched two EST clones, zo50e01 and $\operatorname{tg} 07 \mathrm{a} 01$. The sequences obtained by $3^{\prime}$-RACE from RNA of adult skeletal muscle and fetal brain completely matched those of the two ESTs, while the 5'-RACE gave two forms of PCR products, generated by alternative splicing. Alternative splicing occurred in the $5^{\prime}$-noncoding exons (exons 1-4), and both forms of cDNA contained a putative first methionine codon in exon 5. Therefore, the two transcripts may encode the same protein. Because this novel transcript did not completely match any known genes and it spanned the $14 \mathrm{q}$ breakpoint of the translocation of the pa- tient, we concluded that it is a novel gene and designated it as MIPOL1 (the mirror-image polydactyly gene 1 (GenBank Accession No. AY059470).

MIPOL1 contains a 1326-bp open reading frame spanning the region from the first ATG codon in exon 5 to the termination codon in exon 15 . It is predicted to encode 442 amino acids that have two coiled-coil domains in the $\mathrm{C}$ terminus region. The MIPOL1 protein is homologous in its second coiled-coil domain to a protein, CARD14 (caspase recruitment domain protein 14 ).

\section{Expression of human MIPOL1 and mouse Mipol1}

Northern blot analysis of human MIPOL1 in adult heart, liver, skeletal muscle, kidney, and pancreas, and in fetal kidney revealed a definite but very weak expression (Fig. 2), while gene expression was not detected in adult brain, placenta, or lung. At least three different transcripts $(7 \mathrm{~kb}$, $3 \mathrm{~kb}$, and $2 \mathrm{~kb}$ ) clearly appeared in adult heart. In adult skeletal muscle, the strongest signal was for the $2-\mathrm{kb}$ transcript, which most likely corresponds to the sequence we identified, while we could not isolate the two other, longer transcripts. The whole-mount in situ hybridization of E10.5-E13.5 mouse embryos using mouse Mipoll as a probe did not give signals in specific areas or regions, including the limb bud, but showed diverse signals all over the embryo (data not shown).

Mutation analysis of MIPOL1 in patients with mirror-image polydactyly

The PCR direct sequencing revealed no mutation in any exons or in the intron-exon boundary regions of MIPOL1 in the two patients with disorders similar to MIP.

Genes flanking the 2p23.3 breakpoint

The FISH analysis revealed that a BAC clone (RP11247H16) gave signals on both derivative chromosomes 2
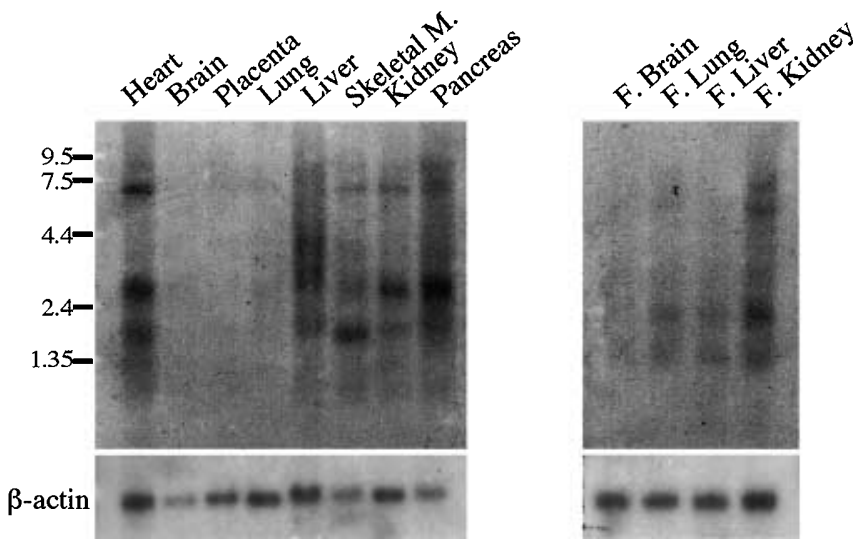

Fig. 2. Northern blot analyses of adult (left) and fetal (right) tissues. Three bands $(7 \mathrm{~kb}, 3 \mathrm{~kb}$, and $2 \mathrm{~kb})$ are clearly visible in the adult heart lane, while the 3-kb signal is dominant in liver, kidney, and pancreas, and the $2-\mathrm{kb}$ signal is dominant in skeletal muscle 
and 14 , in addition to the normal chromosome 2 (data not shown), indicating that the clone covers the $2 \mathrm{p} 23.3$ breakpoint. Because genomic sequences of this clone and overlapped clones are available (GenBank Accession Nos. AC008274, AC010988, and AC008282), we searched for genes and/or ESTs around the breakpoint. We found only one gene, the neuroblastoma-amplified protein gene $(N A G$, GenBank Accession No. NM 015909), which is located at least $50 \mathrm{~kb}$ centromeric to the breakpoint. The $N A G$ gene spans a more than $300-\mathrm{kb}$ genomic region and is transcribed from centromere to telomere. A search for a $200-\mathrm{kb}$ genomic sequence telomeric to the breakpoint found no genes or protein-coding ESTs.

\section{Discussion}

We isolated a novel gene, MIPOL1 (mirror-image polydactyly gene 1$)$, which spanned the $14 \mathrm{q} 13$ breakpoint of $\mathrm{t}(2 ; 14)(\mathrm{p} 23.3 ; \mathrm{q} 13)$ in the patient with MIP (Ohashi et al. 1995; Kim et al. 1997). MIPOL1 in the patient was disrupted between exons 11 and 12 by this translocation, probably leading to a truncated MIPOL1 protein. Our extended gene search in the $1.2-\mathrm{Mb}$ contig found no gene other than MIPOL1 and HNF3A. We also verified the translocation breakpoint on chromosome 2 by FISH analysis. A BAC clone, RP11-247H16, showed split signals on derivative chromosome 2 and derivative chromosome 14 . We performed a computational search around the 2p23.3 breakpoint, but no gene or EST other than $N A G$ was identified. It is possible that there is a gene that was expressed very weakly. $N A G$ is located at least $50 \mathrm{~kb}$ centromeric to the breakpoint and is unlikely to be related to MIP. Thus, it is most likely that MIPOL1 is a strong candidate gene for MIP, although no mutations were detected in two karyotypically normal patients with a similar type of limb anomaly. It remains to be clarified whether other MIP patients have a mutation in MIPOL1, although it is difficult to locate patients with this rare disorder. Since the northern blot analysis disclosed the expression of two other, longer transcripts in human tissues, it also remains to be identified whether these have additional, hidden exons in the $5^{\prime}$ region, and whether the two patients with disorders similar to MIP have a mutation in these putative exons.

The function of MIPOL1 and of its mouse orthologue, Mipoll, is unknown. No gene is known having high sequence homology to these genes. Although mouse Mipoll has not been cloned, its partial EST sequences available from the database show some $85 \%$ homology with those of human MIPOL1. Since human MIPOL1 was expressed very weakly and rather ubiquitously in many tissues examined, except in brain, placenta, or lung, it may have no specific expression profile. A similar result was obtained by whole mount in situ hybridization of Mipoll in E10.5-E13.5 mouse embryos. The MIPOL1 protein has two coiled-coil domains in the C-terminus region. This suggests that MIPOL1 may interact with another protein. CARD14 (caspase recruitment domain protein 14) is a protein that has such a coiled-coil domain (Bertin et al. 2001) with significant homology to the MIPOL1 protein. It has been suggested that the CARD domain of CARD14 plays a role in the phosphorylation of $\mathrm{Bcl10}$ and in the signaling of NF- $\mathrm{\kappa B}$, and its coiled-coil domain may work as the homo- or hetero-oligomerization domain (Bertin et al. 2001). It is thus likely that the second coiled-coil domain of the MIPOL1 protein may work as an oligomerization regulator by comparative protein-protein interaction.

Acknowledgments We thank our patients for their participation. This study was supported by CREST, Japan Science and Technology Corporation, Japan. K.Y. was supported in part by a Grant-in-Aid for Scientific Research (Category C, No. 13203002) from the Ministry of Education, Culture, Sports, Science and Technology of Japan.

\section{References}

Bertin J, Wang L, Guo Y, Jacobson MD, Poyet JL, Srinivasula SM, Merriam S, DiStefano PS, Alnemri ES (2001) CARD11 and CARD14 are novel caspase recruitment domain (CARD)/ membrane-associated guanylate kinase (MAGUK) family members that interact with BCL10 and activate NF-kappa B. J Biol Chem 276:11877-11882

Hatchwell E, Dennis N (1996) Mirror hands and feet: a further case of Laurin-Sandrow syndrome. J Med Genet 33:426-428

Hersh JH, Dela Cruz TV, Pietrantoni M, von Drasek-Ascher G, Turnquest MA, Yacoub OA, Joyce MR (1995) Mirror image duplication of the hands and feet: report of a sporadic case with multiple congenital anomalies. Am J Med Genet 59:341-345

Kantaputra PN (2001) Laurin-Sandrow syndrome with additional associated manifestations. Am J Med Genet 98:210-215

Kim KC, Wakui K, Yamagishi A, Ohno T, Sato M, Imaizumi S, Aihara T, Fukushima Y, Ohashi H (1997) Tetramelic mirror-image polydactyly and a de novo balanced translocation between 2p23.3 and 14q13. Am J Med Genet 68:70-73

Kinoshita A, Tomita H, Makita Y, Yoshida K, Ghadami M, Yamada K, Kondo S, Ikegawa S, Nishimura G, Fukushima Y, Murray JC, Niikawa N, Yoshiura K (2000) Domain-specific mutations in the human transforming growth factor beta 1 gene (TGFB1) result in Camurati-Engelmann disease. Nat Genet 26:19-20

Kogekar N, Teebi AS, Vockley J (1993) Sandrow syndrome of mirror hands and feet and facial abnormalities. Am J Med Genet 46:126128

Laurin CA, Favreau JC, Labelle P (1964) Bilateral absence of the radius and tibia with bilateral reduplication of the ulna and fibula. $\mathrm{J}$ Bone Joint Surg 46-A:137-142

Matsumoto N, Soeda E, Ohashi H, Fujimoto M, Kato R, Tsujita T, Tomita H, Kondo S, Fukushima Y, Niikawa N (1997a) A 12megabase BAC/PAC contig spanning the 14q13 breakpoint of $\mathrm{t}(2 ; 14)$ in a mirror-image polydactyly patient. Genomics 45:11-16

Matsumoto N, Ohashi H, Kato R, Fujimoto M, Tsujita T, Sasaki T, Nakano M, Miyoshi O, Fukushima Y, Niikawa N (1997b) Molecular mapping of a translocation breakpoint at $14 \mathrm{q} 13$ in a patient with mirror-image polydactyly of hands and feet. Hum Genet 99:450-453

Martin RA, Jones MC, Jones KL (1993) Mirror hands and feet with a distinct nasal defect, an autosomal dominant condition. Am J Med Genet 46:129-131

Martinez-Frias ML, Alcaraz M, Espejo P, Gomez MA, Gracia de Leon R, Gonzalez Moro L (1994) Laurin-Sandrow syndrome (mirror hands and feet and nasal defects): description of a new case. J Med Genet 31:410-412

Ohashi H, Kin Y, Iwasaki M, Ohno T, Sato M, Imaizumi S, Aihara T, Yamagishi A, Wakui K, Fukushima Y (1995) Tetramelic mirrorimage-like polydactyly and de novo balanced autosomal translocation [46,XY,t(2;14)(p23.3;q13)]. Am J Hum Genet 57:A98

Yoshiura K, Leysens NJ, Reiter RS, Murray JC (1998) Cloning characterization and mapping of the mouse homeobox gene, Hmxl. Genomics 50:61-68 